\title{
ARTÍGULO ORIGINAL: Evolución de los pacientes con Nefritis Lúpica Proliferativa en el Servicio de Reumatología del Hospital San Juan de Dios que completaron tratamiento de 2 años hasta diciembre de 2010
}

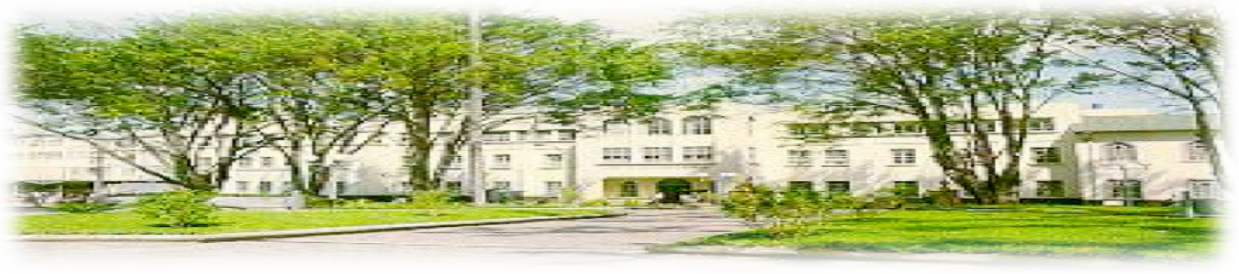

Haspital San Zuan de Dias. San Dasé. Casta Rica. Fundada en 1845

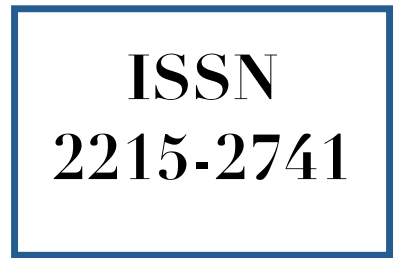

Recibido:

$12 / 04 / 2013$

Aceptado:

$24 / 04 / 2013$

Jorge Carlos Calvo Lon ${ }^{1}$ Alexis Méndez Rodríguez ${ }^{2}$

${ }^{1}$ Médico Especialista en Reumatología. Unidad de Reumatología. Sección de Medicina. Hospital San Juan de Dios.

${ }^{2}$ Médico Residente en Medicina Interna. Programa de Posgrado en Especialidades Médicas UCRCENDEISSS. Correo electrónico: jorge.calvolon@gmail.com

\section{RESUMEN}

Se realizó un estudio observacional y descriptivo de registros médicos, para documentar cuál era el manejo más efectivo para la Nefritis Lúpica Proliferativa en los pacientes del Servicio de Reumatología del Hospital San Juan de Dios. Los pacientes incluidos en el estudio fueron nacionales o extranjeros, sin importar edad, género, etnia o religión; con diagnóstico de Nefritis Lúpica Proliferativa, en control en Reumatología del HSJD, hasta diciembre de 2010 y que completaron el tratamiento de inducción de 2 años. La metodología de revisión fue mediante un instrumento, revisando los expedientes con diagnóstico de Lupus Eritematoso Sistémico. Se revisó 151 expedientes, de los cuales solo 7 reunían los criterios para ser incluidos en el estudio, donde no se pudo realizar una comparación adecuada de los diferentes esquemas de tratamiento y su efectividad.

\section{PALABRAS CLAVE}

Lupus Eritematoso Sistémico. Nefritis Lúpica. Síndrome Nefrótico. Proteinuria. Terapia de Inmunosupresión.

\section{ABSTRACT}

An observational and descriptive study is presented. The main objective was to determine which is the most effective treatment scheme for those patients suffering from Systemic Lupus Erythematous and Lupic Nephritis in San Juan de Dios Hospital, San José, Costa Rica. Every patient with proliferative lupus nephritis was included. There were no restrictions for nationality, religion, gender or age. The only restriction applied was that the patient should complete the 2-years induction therapy. A total of 151 files were revised, but only 7 cases had completed the induction therapy. Because of this fact, the au- 
thor was not able to perform an appropriate comparison with the different treatment schemes found in the revisited medical literature.

\section{KEY WORDS}

Systemic Lupus Erythematosus. Lupic Nephritis. Nephrotic Syndrome. Proteinuria. Immunosupresive Therapy.

\section{INTRODUCCIÓN}

\section{Antecedentes}

La Nefritis Lúpica es una causa importante de morbilidad y mortalidad en los pacientes con lupus eritematoso sistémico ${ }^{(1,2)}$. Es una enfermedad multi sistémica crónica de causa desconocida, heterogénea y con una gran variación de manifestaciones clínicas. Puede ser catalogada como un desorden autoinmune inflamatorio de etiología desconocida.

La presentación clínica de la Nefritis puede tener diferentes variantes, tales como:

- Síndrome Nefrótico y/o Síndrome Nefrítico.

- Hipertensión Arterial.

- Insuficiencia Renal Crónica.

Con respecto a la epidemiología, se anotan los siguientes datos ${ }^{(3,7)}$ :

- Distribución mundial.

- Afecta mayormente la raza negra.

- La incidencia aumenta con la edad.

- Más frecuente en mujeres respecto a hombres con relación 9:1.

- Casi el 90\% pacientes con LES tienen algún grado de nefritis Lúpica

\section{Fisiopatología:}

La Nefritis Lúpica es una complicación del Lupus caracterizada por diferentes mecanismos de ataque al riñón, dentro de los más importantes se encuentra el depósito de complejos inmunes, que llevan a procesos inflamatorios tanto glomerulares como intersticiales, causando daño renal que lleva a insuficiencia renal crónica.
El diagnóstico y clasificación de esta complicación se realiza por medio de biopsia y análisis histopatológico, que permite dividirlo en 6 clases. Esta diferenciación tiene implicaciones en cuanto al manejo general $y$ tratamiento específico.

\section{Definiciones}

- Nefritis Lúpica Proliferativa: Comprende de los clases III y IV de la nefritis lúpica, donde hay lesión endo y/o extracapilar activa o inactiva focal, segmentaria o global, comprometiendo los glomérulos, típicamente con depósitos inmunes subendoteliales, con o sin alteraciones mesangiales

- Proteinuria: Se define micro proteinuria de 30 a $300 \mathrm{mg}$ en orina $24 \mathrm{~h}$, para efectos de proteinuria secundaria a LES se tomó la presencia de más $500 \mathrm{mg}$ en orina de $24 \mathrm{hrs}$.

- Síndrome Nefrótico: Proteinuria (3.5 $\mathrm{g} / \mathrm{d})$, hipoalbuminemia $(<3 \mathrm{~g} / \mathrm{dl})$, edema, hiperlipidemia y lipiduria.

- Hipertensión Arterial: Presión arterial mayor de 139/89 mmHg.

- Remisión Clínica de la Nefritis: Se define como una proteinuria inferior a 0.3 g. en $24 \mathrm{~h}$, con sedimento urinario normal, albuminemia normal y valores de creatinina sérica y aclaramiento endógeno de creatinina por debajo de un $15 \%$ mayor que el valor basal.

- Remisión Parcial de la Nefritis Lúpica: Se define como proteinuria entre 0.3 a $2.9 \mathrm{~g}$ en $24 \mathrm{~h}$, albuminemia de al menos $3.0 \mathrm{~g} / \mathrm{dL}$ y función renal estable.

- Aclaramiento endógeno de creatinina: Es la mejor medición clínica para tener la tasa de filtración glomerular, porque es producido continuamente en el cuerpo y libremente filtrado pero no reabsorbido.

La Nefritis Lúpica Proliferativa es una enfermedad que causa morbilidad y mortalidad importante en los pacientes con Lupus Eritematoso Sistémico, siendo responsable de numerosos días de internamiento y acelerando la insuficiencia renal a su fase terminal. A pesar de no ser la patología de mayor incidencia en la práctica reumatológica, es quizás una de las que genera un gran interés por lo complejo de su 
fisiopatología. Además ha generado diferencias de criterio de en el manejo, existiendo actualmente tanto el esquema del NIH y el grupo del Euro Lupus, así como aquellos que prefieren el uso de ciclofosfamida oral, azatioprina oral y más recientemente los que proponen el uso de micofenolato mofetilo como terapia de inducción.

De ahí la importancia de conocer con mayor detalle el comportamiento de esta patología en nuestros pacientes, con el fin de valorar el esquema de tratamiento con mayor éxito y menor complicación. Un estudio de este tipo nunca se ha realizado en Costa Rica, de modo que se desconoce el esquema de tratamiento más exitoso, para la inducción de la remisión en la Nefritis Lúpica Proliferativa, así como las complicaciones más comunes asociadas.

Tabla 1. Tratamiento de la Nefritis Lúpica según sus características clínicas y anatomopatológicas

\begin{tabular}{|c|c|c|c|}
\hline $\begin{array}{l}\text { Clasificado por } \\
\text { biopsia }\end{array}$ & Inducción & Mantenimiento & Terapia Adyuvante \\
\hline Cambios Mínimos & $\begin{array}{l}\text { Prednisona } 0.5 \mathrm{mg} / \mathrm{Kg} / \mathrm{día} / 4 \\
\text { semanas }\end{array}$ & $\begin{array}{l}\text { Reducción progresiva de los } \\
\text { GC }\end{array}$ & Control estricto del PA y cLDL \\
\hline Mesangial & $\begin{array}{l}\text { Prednisona } 0.5 \mathrm{mg} / \mathrm{Kg} / \mathrm{día} / 4 \\
\text { semanas }\end{array}$ & $\begin{array}{l}\text { Reducción progresiva de los } \\
\text { GC }\end{array}$ & Control estricto del PA y cLDL \\
\hline Proliferativo focal & $\begin{array}{l}\text { Prednisona } 0.5 \mathrm{mg} / \mathrm{Kg} / \mathrm{dí} / 4 \\
\text { semanas + CF o MMF }\end{array}$ & MMF o AZA & Control estricto del PA y cLDL \\
\hline Proliferativa Difusa & $\begin{array}{l}\text { Prednisona } 0.5 \mathrm{mg} / \mathrm{Kg} / \mathrm{dí} / 4 \\
\text { semanas + CF o MMF }\end{array}$ & MMF o AZA & Control estricto del PA y cLDL \\
\hline $\begin{array}{l}\text { Proliferativo focal o } \\
\text { Difuso de mal } \\
\text { pronóstico* }\end{array}$ & $\begin{array}{l}\text { Prednisona } 1 \mathrm{mg} / \mathrm{Kg} / \text { día } / 4 \\
\text { semanas + CF }\end{array}$ & MMF o AZA & Control estricto del PA y cLDL \\
\hline Membranosa & $\begin{array}{l}\begin{array}{l}\text { Prednisona } \\
\text { semanas }\end{array} \\
\text { mg/Kg/día } / 4\end{array}$ & $\begin{array}{l}\text { Reducción progresiva de los } \\
\text { GC. En caso de resistencia } \\
\text { o dependencia a GC, } \\
\text { valorar CF o MMF o CsA }\end{array}$ & Control estricto del PA y cLDL \\
\hline Esclerosis & No Precisa & No Precisa & Control estricto del PA y cLDL \\
\hline
\end{tabular}

AZA: azatriopina. CF: ciclofosfamida. cLDL: colesterol ligado a las lipoproteínas de baja densidad. CsA: ciclosporina A. GC: glucocorticoides. MMF: micofenolato mofetilo. PA: presión arterial. *Insuficiencia Renal o Proteinuria en rango nefrótico o raza negra o hipertensión arterial de difícil control.

Fuente: modificado de (3)

\section{Pregunta de investigación}

Cuál es la evolución en el manejo de los pacientes con Nefritis Lúpica Proliferativa en lo que concierne tasas de remisión $y$ complicaciones?

\section{Objetivo General}

Conocer la evolución de los pacientes diagnosticados con Nefritis Lúpica Proliferativa en el servicio de reumatología del HSJD.

\section{Objetivos Específicos}

- Identificar los diferentes esquemas de tratamiento para la Nefritis Lúpica Proliferativa.
- Identificar el éxito de remisión según los esquemas de tratamiento de Nefritis Lúpica Proliferativa en estos pacientes.

- Identificar cuáles son las complicaciones infecciosas presentadas por los pacientes con Nefritis Lúpica Proliferativa en control en Reumatología del HSJD.

- Determinar el tiempo transcurrido para el desarrollo de complicaciones y evolución de las mismas. Identificar la procedencia de los pacientes portadores de Nefritis Lúpica, así como otros aspectos epidemiológicos importantes tales como distribución por sexo, edad, ocupación, edad al diagnóstico y tiempo de evolución. 


\section{Metodología}

Diseño del estudio: Observacional descriptivo transversal retrospectivo.

Población de Estudio: Se trabajó con toda la población de pacientes con LES, nacionales o extranjeros, sin importar edad, género, etnia o religión; con diagnóstico de Nefritis Lúpica Proliferativa y que se encontraban en control en Reumatología del HSJD hasta diciembre de 2010. Además, los pacientes debían haber completado el tratamiento de inducción por 2 años.

\section{Criterios de Inclusión}

Pacientes con Nefritis Lúpica Proliferativa en control en Reumatología del HSJD hasta Agosto del 2010.

\section{Criterios de Exclusión}

- Pacientes con diagnóstico de Nefritis Lúpica Proliferativa diagnosticados inicialmente en HSJD y sigan su control en otro hospital.

- Pacientes portadores de Nefritis Lúpica sin confirmación histológica.

- Pacientes con Nefritis Lúpica no proliferativa.

- Pacientes con Nefritis Lúpica Proliferativa que abandonaron su tratamiento.

\section{Variables del estudio}

- Edad: variable cuantitativa discreta. Se determinó la edad en años del paciente al momento del diagnóstico de la enfermedad y la edad actual.

- Procedencia: variable cualitativa nominal.

- Sexo: variable cualitativa nominal.

- Ocupación: variable cualitativa nominal. Se estableció la ocupación del paciente al momento del diagnóstico de la Nefritis Lúpica Proliferativa.

- Comorbilidad (complicaciones): variable cualitativa. Se documentó la presencia de complicaciones asociados a los diferentes esquemas de tratamiento.

- Métodos diagnósticos: variable cualitativa. Se determinó el grado de
Nefritis Proliferativa mediante biopsia renal.

- Estudios de Laboratorio: variable cuantitativa. Se determinó la evolución del paciente mediante examen de orina de 24 horas, albúmina en sangre y pruebas de función renal.

- Tratamiento médico: variable cualitativa. Se documentó los diferentes esquemas terapéuticos empleados en el manejo de estos pacientes.

- Tiempo trascurrido para la remisión de la enfermedad, mediante pruebas de laboratorio al diagnóstico, al mes, a los 6 meses y a los 2 años.

- Evolución clínica del paciente: variable cualitativa que incluyó si el paciente entró en remisión (proteinuria inferior a $0.3 \mathrm{~g}$. en $24 \mathrm{~h}$, con sedimento urinario normal, albuminemia normal y valores de creatinina serica y aclaramiento endógeno de creatinina por debajo de un $15 \%$ mayor que el valor basal), mantiene la enfermedad activa, las complicaciones que sufre durante el periodo de tratamiento.

\section{Análisis Estadístico}

Las variables se tabularon inicialmente en una hoja de cálculo Excel (Microsoft ${ }^{\odot}$ 2003) y se analizaron en Epi Info 3.3.2 (CDC-2005). Se utilizó métodos de estadística descriptiva, medidas de tendencia central y asociación de variables. Se realizó una tabulación de datos mediante hoja recolectora adjunta.

\section{RESULTADOS}

De los 151 pacientes con diagnóstico de Lupus Eritematosos Sistémico del Servicio de Reumatología del Hospital San Juan de Dios, 36 pacientes tenían el diagnostico de Nefritis Lúpica como parte de sus diagnósticos. De éstos, solamente 7 pacientes cumplieron con los criterios de inclusión.

Los esquemas de tratamiento utilizados fueron:

- 4 casos con ciclofosfamida IV + esteroides

- 2 pacientes con azatrioprina oral + esteroides 
- 1 caso de ciclofosfamida oral + esteroides.

En cuanto al éxito de los esquemas que se utilizaron, éstos no pueden ser comparados, ya que el tamaño de la muestra generada en el estudio no es estadísticamente significativa para poder dar conclusiones sobre la eficacia de un tratamiento comparándolo con otro, sin embargo se encontró que de los 4 casos manejados con ciclofosfamida IV + esteroides, 3 lograron remisión y 1 paciente permaneció con la proteinuria y pérdida de su función renal después de 2 años.

Los 2 casos manejados con azatrioprina oral + esteroides entraron en remisión de su después de 2 años.

El único casos manejado con ciclofosfamida oral + esteroides entró en remisión después de 2 años.

Ninguno de los casos que cumplió con los criteros de inclusión presentó complicación infecciosa alguna.

No se pudo realizar comparación y tabulación de las complicaciones ya que no hubo ninguna documentada en los expedientes que se revisaron.

En cuanto a la edad en años de diagnóstico de nefritis lúpica, fue: 42, 27, 24, 22, 23, 27 y 32; siendo así el rango de 20 a 42 años el más afectados. La edad de diagnóstico de LES no se pudo documentar, ya que no se anotó como tal en los expedientes.

\section{DISCUSIÓN}

El valor estadístico de los resultados obtenidos es pobre, ya que la muestra es pequeña. Por otro lado, el diagnóstico de la entidad estudiada se dificulta por la necesidad de realizar una biopsia renal para confirmarlo.

Otra limitación que se encontró al revisar los expedientes, fue que no todos los pacientes cumplen con los ciclos de tratamiento de inducción y continuación en la evolución de su enfermedad, por lo cual se dificultó documentar los verdaderos portadores de esta patología y realmente cuál es el método de tratamiento más eficaz.
Por otro lado, se imposibilita realizar un adecuado revisión para transmitir los verdaderos resultados que obtenemos con los diferentes tratamientos para la Nefritis Lúpica Porliferativa, ya que muchos pacientes con este diagnóstico no tienen biopsia renal, que es clave para determinar el estadio de la enfermedad.

Algunos pacientes con biopsia renal y que recibieron tratamiento, no tuvieron seguimiento por varias razones, por ejemplo que el paciente se trasladara a otro centro para su control y abandono de la consulta. También hubo un grupo de pacientes con biopsia que no pudo ser incluído, debido a que la clasificación histológica no fue la de Nefritis Lúpica Proliferativa.

Algunos de los expedientes solicitados también estaban extraviados y no se pudieron revisar.

La iniciativa de realizar estudios retrospectivos, de revisión de expedientes clínicos puede toparse con estas dificultades que no permiten realizar un estudio adecuado y en este caso va desde la falta de documentación en los expedientes, realizar protocolos de diagnóstico acertados para cada enfermedad y poder dar el seguimiento a los pacientes hasta ver la remisión, recaida $o$ persistencia de la enfermedad.

La principal recomendación del presente estudio es una llamada a todo el personal médico, sobre la importancia del expediente médico, la documentación adecuada, seguir protocolos de diagnóstico y seguimiento, para el manejo y documentación adecuado del paciente, que permitan realizar estudios precisos para alcanzar conclusiones apropiadas, aplicables al resto de la población.

\section{CONCLUSIONES Y RECOMENDACIONES}

Los objetivos del estudio se alcanzaron parcialmente, ya que la cantidad de pacientes que cumplió con los criterios de inclusión fueron muy pocos y no se pudo obtener una muestra estadísticamente significativa, que representara la cantidad de casos portadores de esta patología.

No se pudo hacer una comparación real de los diferentes tratamientos y los resultados obtenidos, para así determinar cuál es el más efectivo y que produce menos efectos secundarios. 


\section{REFERENCIAS BIBLIOGRÁFICAS}

1. Tang S Liu S Lai K. Pathogenesis of Lupus Nephritis: An Update. Nephrology 2005;10:174-179.

2. Davidson A Aranow C. Pathogenesis and treatment of Systemic Lupus Erythematosus Nephritis. Current Opinion in Rheumatology 2006;18:468-475.

3. Fernández L Adreu J Ginzler E. Tratamiento de la nefritis lúpica. Reumatol Clin. 2008;4(4):140-151.

4. Weening J D'Agati V Schwartz M. The Classification of Glomerulonephritis in Systemic Lupus Erythematosus Revisited. J Am Soc Nephrol 2004;15:241250.

5. Cameron JS. Lupus Nephritis. J Am Soc Nephrol 1999;10:413-424.

6. Houssiau F. Management of Lupus Nephritis: An Update. J Am Soc Nephrol 2004;15:2694-2704.

7. Balow JE Austin HA III. Treatment of Proliferative Lupus Nephritis. American Journal of Kidney Diseases. 2004;43(2):383-385

8. Lau K Wyatt R. Glomerulonephritis. Adolesc Med 2005;16:67-85.

9. Flank RS Roberts MA Stripoli G. Treatment of Diffuse Proliferative Lupus Nephritis: A Meta-Analysis of Randomized Controlled Trials. American Journal of Kidney Diseases, 2004;43(2):197-208.

\section{CONFLICTO DE INTERÉS}

En el presente trabajo no existió ningún conflicto de interés. 